all patients at all times. Left ventricular function remained unchanged in the ischemic group.

Conclusion: In patients with IHD, prolonged and repeated ischemic training sessions up to $60 \mathrm{~min}$ can be well tolerated without evidence of myocardial injury, significant arrhythmias or left ventricular dysfunction. Thus exercising at or above the ischemic threshold does not appear deleterious under this kind of supervision.

Supported by Institut de cardiologie de Québec, Inc.

\section{Taking the Stress Out of Stress Testing}

Hohenleitner Miller, Rosemary, Pennsylvania Hospital, Philadelphia, PA, USA

Background: Stress testing is a widely used screening tool for finding coronary artery disease, the leading killer of men and women in the United States. Stress tests are a safe noninvasive way to detect coronary ischemia and sift out which patients may benefit from percutaneous coronary intervention or coronary artery bypass graft. This presentation is an overview of the factors to be considered when Stress Testing.

Methods: Many factors must be considered before deciding which type of stress test best suits the patient. Time, cost and patient compliance as well as preexisting factors in their medical history such as their electrocardiogram, pacemaker or internal defibrillator, and medications. Keep in mind the following conditions make the EKG portion uninterpretable: LBBB, LVH with strain, repolarization abnormalities secondary to digitalis, baseline abnormalities, and ventricular pacing. If patients are not fit enough to perform an adequate amount of time on a treadmill due to deconditioning or musculoskeletal problems such as arthritis, a pharmacologic stress test should be performed such as a Persantine or Adenosine stress test. When patients are taking heart lowering medication like beta blockers, non-hydropyridine calcium channel blockers, digitalis, or amiodarone, they may have a blunted heart rate response making the EKG portion of the test non-diagnostic. Such patients can be instructed not to take their medication that morning or may consider having a pharmacalogic test instead.
Results: Viability studies are sometimes coupled with pharmacalogic stress tests to see if the cardiac muscle is viable and would benefit from perfusion by percutaneous intervention or bypass. These tests are performed by giving the patient sublingual nitroglycerin prior to performing the resting images in a nuclear stress test.

Conclusion: Many factors must be taken into consideration before ordering a stress test to see which one would benefit the patient the most. Keep in mind the patients physical condition, medications, and baseline EKG before choosing which one to order. 